\title{
Diagnosis of male hypogonadism: what is low testosterone
}

Moises Mercado

Experimental Endocrinology Unit, Hospital de Especialidades, CMN, S.XXI, IMSS, Mexico City, Mexico

Address for correspondence:

Moisés Mercado, MD, FRCP(C)

Experimental Endocrinology Unit

Hospital de Especialidades, CMN, S.XXI, IMSS

Aristóteles 68, Polanco

11560 Mexico City, Mexico

E-mail: mmercadoa@yahoo.com

\section{Summary}

In contrast to primary hypogonadism, whereby the rise of gonadotropin levels helps establishing a diagnosis, the diagnosis of secondary hypogonadism relies for the most part, on the identification of a low testosterone level in the right clinical context. Establishing that a patient indeed has a low testosterone level requires taking into account several physiological as well analytical aspects. The vast majority of testosterone in men circulates in plasma bound to albumin $(50 \%)$, sex-steroid binding globulin (SHBG) (44\%) and other proteins $(4 \%)$, while only $2 \%$ is found free. The biologically active fraction consists of the free and the albumin-bound fractions. Testosterone levels are highest in the early morning and lowest during the summer. Both total and free testosterone decline with age, as SHBG levels increase. Conditions such as obesity and diabetes also result in diminished testosterone concentrations, while they are accompanied by low SHBG levels. Currently, measurement of total testosterone in hospital laboratories is usually performed on fully automated immunoassay analyzers. Although tandem mass spectrometry methods after gas or liquid chromatography are the most accurate means for testosterone measurement, they are still not widely available in most clinical laboratories. In some cases the concentration of total testosterone may not always represent a true reflection of the patient androgen status and therefore an estimate of the non-SHBG or bioavailable fraction may be a more appropriate measure. Methods of assessment of the non-SHBG-bound fraction of testosterone include estimation of the free concentration by methods including the calculation of the free androgen index (FAI), equilibrium dialysis, centrifugal ultrafiltration, direct analog RIA or calculation of the free fraction.

KEY WORDS: testosterone; free testosterone; SHBG; hypogonadism.

\section{Introduction}

Testosterone is the main androgen synthesized and secreted by the normal adult male testicle $(1,2)$. The regulation of the gonadal axis begins at hypothalamic nuclei where highly specialized neurons "awake" during puberty and start synthesizing and releasing $\mathrm{GnRH}$ (Gonadotrophin releasing hormone) in a pulsatile manner, although higher brain nuclei and sites including the neocortex, also play an important role $(1,2)$ (Fig. 1). $\mathrm{GnRH}$ acts through specific Gs-protein coupled membrane receptors on the gonadotroph cells of the anterior pituitary, resulting in the transcription of the genes that encode the specific beta subunits of $\mathrm{LH}$ and FSH as well as the common alpha subunit, and subsequently in the secretion of these gonadotropins into the systemic circulation $(1,2)$ (Fig. 1). LH exerts its biological effects, stimulating the testosterone-producing Leydig cells, whereas FSH targets the Sertoli cells and is more involved in spermatogenesis as well as in the synthesis and secretion of inhibin $(1,2)$ (Fig. 1). The testosterone secreted by the testicle, in turn, inhibits the release of $\mathrm{LH}$ and $\mathrm{FSH}$ via a negative feedback loop at both the hypothalamic and pituitary levels,

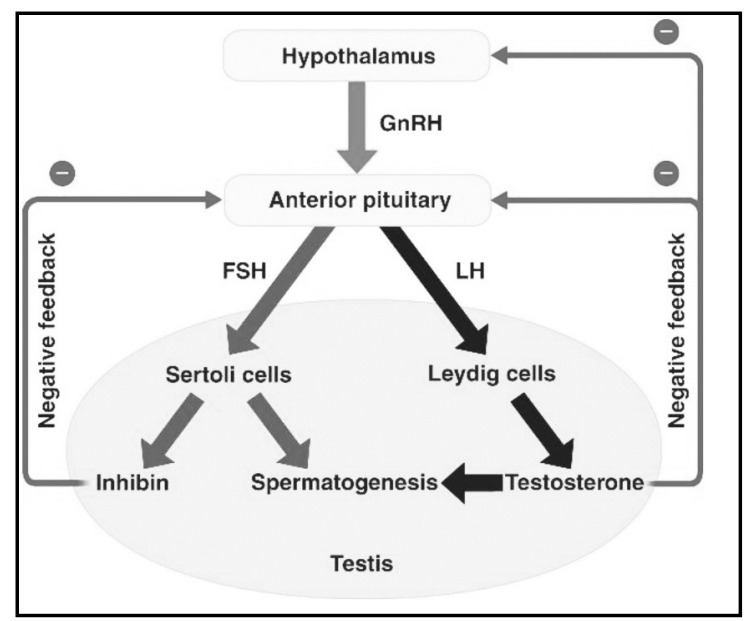

Figure 1 - Regulation of the male hypothalamic-pituitarytesticular axis. 
Table 1 - Etiology of primary male hypogonadism.

\begin{tabular}{ll}
\hline \multicolumn{2}{c}{ Primary Male hypogonadism } \\
\hline Congenital & Acquired \\
- Klinefelter Syndrome & - Mump.s and other \\
- FSH/LLH receptor & infections \\
mutations & - XRT and alkylating agents \\
- Varicocele & - Ketoconazole \\
- Cryptorchidism & - Glucocorticoids \\
- Disorders of androgen & - Environmental toxins \\
synthesis & - Trauma \\
- Myotonic dystrophy & - Testicular torsion \\
- Acquired & - Autoimmune \\
& - Hepatic \& renal failure \\
& - HIV \\
\hline
\end{tabular}

Table 2 - Etiology of secondary male hypogonadism.

\begin{tabular}{ll}
\hline \multicolumn{2}{c}{ Secondary Male Hypogonadism } \\
\hline Congenital & Acquired \\
- Kallman syndrome & - Suppression of \\
- DAXI and GPR54 & gonadotropins \\
mutations & - Hyperprolactinemia \\
- Leptin and leptin rec. mut. & - Gonadal steroids \\
- Prader-Willy syndrome & glucorticoid adm. \\
- Gonadotropin SU & - Opiates \& GnRH analogs \\
mutations & - Critical illness \\
- Pituitary cytodifferentiation & - Chronic systemic \\
abnormalities & disease \& DM \\
& - Gonadotrope damage \\
& - Pituitary adenomas \\
& - Craneopharyngiomas \\
& - Pituitary mets \\
& - Pituitary apoplexy \\
& - Trauma \\
& - Surgery and XRT to the \\
& sellar region \\
\hline
\end{tabular}

whereas inhibin acts only at the pituitary level $(1,2)$ (Fig. 1). Irrespective of the etiology, in primary hypogonadism the loss of testosterone negative feedback results in a significant increment in gonadotropin secretion $(1,2)$ (Tab. 1). In contrast, gonadal failure or insufficiency resulting from pituitary or hypothalamic causes is associated with low or inappropriately normal serum concentrations of LH and FSH $(1,2)$ (Tab. 2). Thus whereas the diagnosis of primary hypogonadism is facilitated by the finding of high gonadotrophins in blood, the biochemical diagnosis of secondary hypogonadism relies only on the measurement of serum testosterone in the appropriate clinical context. Establishing that a patient has a low testosterone level is not always an easy task since several physiological and analytical aspects need to be taken into consideration. The purpose of this review is to summarize such aspects.

\section{Basic physiology and biochemistry of circulating testosterone in men}

The vast majority of testosterone in the adult male circulates in plasma bound to albumin (50\%) and sex hor- mone binding globulin (SHBG, $44 \%$ ), while only $2 \%$ is found free $(3,4)$. The biologically active fraction consists of the free and the albumin-bound fractions $(3,4)$. The precise role of these specific binding proteins remains incompletely understood. However, SHBG in particular appears to actively participate in the transport of testosterone into target tissues like the prostate and the testes themselves (5). Like with other steroid hormones such as estradiol and cortisol, the rise in binding proteins is associated with a concomitant increment in the measured total testosterone concentrations $(3,4)$.

Testosterone secretion follows a circadian rhythm with the highest level found in the morning and the lowest in the evening (6). Such diurnal variation is clearly seen in males up to the seventh decade and appears to be distorted thereafter (6). The existence of a seasonal variation is controversial but it has been suggested that testosterone concentrations are higher during the winter and lower during the summer (6). Testosterone levels decrease progressively from early adulthood to older age (7-9). This is particularly true for free testosterone as SHBG concentrations rise with advancing age (7-9). The decline of testosterone with age is profoundly influenced by adiposity and the concomitant increased prevalence of conditions like diabetes that are also associated with low androgen concentrations (79). In contrast to menopause in women, andropause in men is more of a continuous age-dependent process with high inter-individual variability and overlapping serum testosterone concentrations between age groups (7-9). Serum concentrations of total testosterone are lower in obese men and this is for the most part due to a concomitant reduction in SHBG levels (7). Acute illness, HIV infection and malnutrition are associated with low total and free testosterone concentrations due to an adaptive state of secondary hypogonadism (7).

\section{Analytical aspects of testosterone measurement}

Total testosterone:

Testosterone measurement methods suffer from a lack of age- and gender-adjusted normal ranges and a universally recognized calibrating standard (10). Most clinical laboratories measure total testosterone (protein-bound plus free testosterone) by fully automated immunoassay analyzers $(7,10,11)$. Prior to immunoassay, binding proteins need to be displaced from testosterone, be it by extraction of the steroid into an organic solvent or by the addition of a chemical agent with a higher affinity for the binding protein $(7,10,11)$. The concentration of total testosterone in normal healthy men is at least four times higher than in normal adult women, and in most laboratories the quoted normal reference values range from 11 to $35 \mathrm{nmol} / \mathrm{L}(7,10,11)$. Compared to the original "manual" RIA, the currently in-use immunoassay platforms are capable of measuring many samples at the same time and allow for a quick turn-around of results $(7,10,11)$. Inter-assay coefficients of variation among total testosterone im- 
munoassays range from 2 to $8 \%(7,10,11)$. The main cross-reactant in testosterone assays is 5-alpha-dihydrotestosterone (DHT), which can still be detected after solvent extraction. However, the normal concentrations of serum DHT are considerably lower than those of testosterone and are rarely elevated enough to significantly interfere with testosterone measurement (7). Abnormally high testosterone concentrations ( $>40$ $\mathrm{nmol} / \mathrm{L}$ ) are usually only found in patients receiving replacement therapy, since testosterone-secreting tumors are very rare. Spuriously low testosterone concentrations due to analytical causes are also very infrequent but can be found when the binding proteins in the sample are not properly extracted or even more rarely, by autoantibodies that recognize the labeled antigen $(7,10,11)$.

Direct immunoassays without prior extraction for binding proteins tend to overestimate testosterone levels and have limited accuracy at concentrations below 11 $\mathrm{nmol} / \mathrm{L}(7,10,11)$. Although these direct assays are currently fully automated and provide a rapid turnaround time, they are susceptible to matrix effects and the reference intervals are not standardized and totally method-dependent $(7,10,11)$. RIA after chromatography extraction has been extensively used and there are well-documented reference intervals in different populations, however, it is technically cumbersome and very few clinical laboratories are qualified to do it $(7,10,11)$. Mass spectrometry (MS) after extraction with either liquid or gas chromatography is highly accurate when properly validated and allows measurement of multiple steroids in the same aliquot, based on their chemical structures; tandem MS can increase the specificity of the method $(12,13)$. The drawbacks of MS are their relatively high cost, lack of standardization and limited throughput (10).

Free testosterone:

As mentioned before, less than $2 \%$ of circulating testosterone is found free in plasma; the term bioavailable testosterone refers to the free and albumin-bound fractions, and appears to be biologically active. The reference methods for measuring free testosterone are equilibrium dialysis and centrifugal ultrafiltration $(14,15)$. Both of these methodologies are technically cumbersome and thus, not widely available in clinical laboratories. Free testosterone is measured at many hospital laboratories by means of a tracer analog technique or direct RIA, which are rather inaccurate and non-validated (10). Bioavailable testosterone is measured in reference laboratories by the ammonium sulfate precipitation technique, which is also technically difficult (16).

Methods for estimating free testosterone:

In view of the difficulties in measuring free testosterone by robust and reliable methods such as equilibrium dialysis, several equations have been developed to estimate the percent of free testosterone in serum. Besides the total testosterone concentration, these formulae require the accurate measurement of SHBG concentrations, which can be done by immunoassays.
The simplest of these equations calculates the free androgen index (FAl) as follows: $\mathrm{FAl}=[$ Total testosterone] $x$ 100/[SHBG].

The FAl is useful in the diagnosis of hyperandrogenism in women but is not very reliable in discriminating hypogonadal from normal men (7). Another, more accurate way of calculating free testosterone based on the law of mass action also uses the measured total testosterone, SHBG and albumin but includes in the equation the dissociation constants $(\mathrm{Kd})$ for testosterone with SHBG and with albumin $(14,17)$. This method correlates very well with free testosterone measured by equilibrium dialysis $(14,17)$. These and other algorithms for the calculation of free and bioavailable testosterone are available at the website of the International Society for the Study of the Ageing Male (www.issam.ch).

\section{Prevalence of androgen deficiency in men}

Late-onset hypogonadism is a clinical and biochemical state with advancing age, characterized by particular symptoms and a low testosterone level (10). The exact prevalence and incidence of this syndrome in men is not known, as most of the published epidemiological studies used poorly validated immunoassay measurements of total testosterone (10). Such studies have used different definitions of hypogonadism based on the presence of symptoms, but the majority simply reports the prevalence of low testosterone levels $(8,10)$. Thus, the precise testosterone level below which, symptoms of androgen deficiency emerge and adverse health outcomes ensue remains unclear.

Relatively recent publications of the European Male Aging Study (EMAS) provide a reasonable approximation to solving this issue (18-20). The EMAS has analyzed data from almost 4000 middle age and elderly men, recruited at 8 European centers (18-20). A crosssectional analysis of over 3000 men spanning 4 decades of age from 40 to 80 years old found a decline in total and free testosterone concentrations of $8.6 \%$ and $33.1 \%$, respectively, along with a $47 \%$ rise in SHBG levels (18). In this study, multiple factors altering the gonadotropic axis, such as obesity, smoking and the presence of co-morbidities like diabetes, are superimposed on the progressive testicular impairment associated with age (18). More recent reports from the same group, linked specific total testosterone-measured by GC-MS/MS-and calculated free testosterone levels with the appearance of symptoms of androgen deficiency $(19,20)$. A decrease frequency of sexual thoughts, erectile dysfunction, a decreased frequency of morning erections and a reduced vigor were associated with total testosterone thresholds of $8,8.5,11$ and $13 \mathrm{nmol} / \mathrm{L}$, respectively; free testosterone levels of 160 $\mathrm{pmol} / \mathrm{L}$, were associated with a decreased frequency of sexual thoughts, whereas erectile dysfunction and decreased frequency of morning erections were linked to a free testosterone level of $280 \mathrm{pmol} / \mathrm{L}(19-20)$. Interestingly, psychological symptoms were not associated with a particular testosterone threshold. In this cohort, 
$4.1 \%$ and $17 \%$ of the population had total testosterone levels below 8 and $11 \mathrm{nmol} / \mathrm{L}$, respectively $(19,20)$. Overall, the prevalence of late-onset hypogonadism was estimated to be $2.1 \%$, ranging from $0.1 \%$ in men aged $40-49$ years, to $5.1 \%$ in men aged $70-79$ years $(19,20)$. Data from the Massachusetts Male Aging Study indicate that the prevalence of symptomatic androgen deficiency is between 6 and $12 \%(21,22)$. A similar figure has been reported in a population-based study derived from the Boston Area Community Health Survey and including Black, Hispanic and White men (23).

Another aspect that needs to be considered is the type of population studied, since the prevalence of androgen deficiency seems to be higher among men attending a primary care facility than among communitydwelling individuals $(24,25)$. The Hypogonadism in Males Study estimated the prevalence of clinical and biochemical androgen deficiency in over 2000 men aged more than 45 years, visiting primary care practices in the United States (24). In this study, the prevalence of hypogonadism was close to $40 \%$; conditions such as diabetes, hypertension, obesity, dyslipidemia and obstructive pulmonary disease, significantly increased the likelihood of androgen deficiency with odds ratios that ranged from 1.5 to 2.4 (24). Androgen deficiency is considerably more common in patients with diabetes. Biswass et al., have recently evaluated 115 subjects with type 2 and 93 with type 1 diabetes and compared them to an age-matched control group (26). These authors found that $45 \%$ and $61 \%$ of their patients with type 2 diabetes had very low levels of total $(<11.9 \mathrm{nmol} / \mathrm{L})$ and free testosterone ( $<260 \mathrm{pmol} / \mathrm{L})$, respectively (26). The prevalence of a low androgen level was considerably lower among patients with type 1 diabetes, yet still significantly higher that in the control population (26). The frequency of low total and free testosterone in this study was strongly affected by age and central adiposity yet, androgen levels correlated rather weakly symptoms of sexual dysfunction and diabetes-related quality of life (26)

\section{Concluding remarks}

- Establishing the diagnosis of androgen deficiency in adult males can be a difficult task due to both physiological and analytical complexities of testosterone measurement.

- The initial hormonal evaluation of a man with probable hypogonadism should include measurement of early morning total testosterone, as well as $\mathrm{LH}$, $\mathrm{FSH}$ and prolactin in order to differentiate primary from secondary hypogonadism. SHBG levels should also be measured in order to be able to estimate free testosterone levels.

- Ideally, total testosterone should be measured by tandem MS after extraction by either gas or liquid chromatography. Alternative methods include automated immunoassays that use an extraction step.

- A total testosterone value below $8 \mathrm{nmol} / \mathrm{L}$ is indicative of androgen deficiency and correlates well with the presence of symptoms. In subjects with values between 8 and $11 \mathrm{mmol} / \mathrm{L}$, the free testosterone level is helpful in establishing a diagnosis.

- The prevalence of androgen deficiency among aging males varies according to the type of population studied. Among community dwelling populations it is reported to be between 6 and $12 \%$, whereas among patients attending primary care facilities it can be as high as $30-40 \%$.

- Normal aging is associated with a decline in testosterone levels, particularly in the free fraction, but symptoms of androgen deficiency such as sexual dysfunction do not always correlate with hormonal data.

- Conditions such as diabetes, obesity and insulin resistance are also associated with a reduced concentration of testosterone.

- The evaluation of the androgen status should be always carried out considering the clinical context.

\section{References}

1. Reyes Fuentes A, Veldhuis JD. Neuroendocrine physiology of the normal male gonadal axis. Endocrinol Metab Clin North Amer 1993; 22:93-124.

2. Rey RA, Grinspon RP, Gottlieb S, et al. Male hypogonadism: an extended classification based on a developmental, endocrine physiology-based approach. Andrology 2013; 1:3-16.

3. Rosner W. Sex steroids and the free hormone hypothesis. Cell 2006; 124:455-456.

4. Dunn JF, Nisula BC, Rodbord D. Transport of steroid hormones:binding of 21 endogenous steroids to both, testosterone binding globulin and corticosteroid binding globulin in human plasma. J Clin Endocrinol Metab 1981; 53:58-68.

5. Hammond GJ. Diverse roles for sex hormone binding globulin in reproduction. Biol Reprod 2011; 85:431-441.

6. Brambilla DJ, Matsumoto AM, Araujo AB, McKinlay $J B$. The effect of diurnal variation on clinical measurement of serum testosterone and other sex hormone levels in men. J Clin Endocrinol Metab 2009; 94:907:913.

7. Diver MJ. Analytical and physiological factors affecting the interpretation of serum testosterone concentration in men. Ann Clin Biochem 2006; 43:3-12.

8. Diver MJ, Imtiaz KE, Ahmad AM. Diurnal rhythms of serum total, free and bioavailable testosterone and sex hormone binding globulin in middle-aged men compared with those in young men. Clin Endocrinol 2003; 58:710-718.

9. Leifke E, Herenoi V, Wichus C, et al. Age-related changes of serum sex hormones, insulin-like growth factor-1 and sex hormone binding globulin levels in men:cross-sectional data from a healthy male cohort. Clin Endocrinol 2000; 53:689-695.

10. Rosner W, Auchus RJ, Azziz R, et al. Utility, limitations and pitfalls in measuring testosterone: An Endocrine Society Position Statement. J Clin Endocrinol Metab 2007; 92:405-413.

11. Matsumoto AM, Bremner AL, Roberts $\mathrm{WL}$, et al. 
Serum testosterone assays-accuracy matters. J Clin Endocrinol Metab 2004; 89:520-524

12. Kushnir MM, Rockwood AL, Roberts WL, et al. Performance characteristics of a tandem mass spectrometry assay for serum testosterone. Clin Chem 2006; 52:120-128.

13. Wang C, Catlin DH, Demers LM, et al. Measurement of total serum testosterone in adult men: comparison of current laboratory methods versus liquid chromatography tandem-mass spectrometry. J Clin Endocrinol Metab 2004; 89:534-543.

14. Vermeulen A, Verdonck L, Kaufman JM. A critical evaluation of simple methods for the estimation of free testosterone in serum. J Clin Endocrinol Metab 1999; 84:3666-3672.

15. Van Uyfanghe K, StockI D, Kaufman JM, et al. Evaluation of a candidate reference measurement procedure for serum free testosterone based on ultrafiltration and isotope-dilution gas chromatography mass spectrometry. Clin Chem 2004; 50:2101-2110.

16. Emadi-Konjin P, Bain J, Bromberg IL. Evaluation of an algorithm for calculation of serum bioavailable testosterone (BAT). Clin Biochem 2003; 36:591-596.

17. Ly LP, Handelsman DJ. Empirical estimation of free testosterone from testosterone and sex hormone binding globulin immunoassays. Eur $\mathrm{J}$ Endocrinol 2005; 152:471-478.

18. Wu FCW, Tajar A, Pye SR, et al. Hypothalamic-pituitary-testicular axis disruptions in older men are differentially linked to age and modifiable risk factors: The European Male Ageing Study. J Clin Endocrinol Metab 2008; 93:2737-2745.

19. Tajar A, Forti G, O'Neil TW, et al. Characteristics of secondary, primary and compensated hypogonadism in aging men: Evidence from the European Male Ageing Study. J Clin Endocrinol Metab 2010;95: 1810-1818.

20. Wu FCW, Tajar A, Beynan JM, et al. Identification of late-onset hypogonadism in middle-aged and elderly men. N Engl J Med 2010;363:123-135.

21. Araujo $A B, O$ 'Donnell $A B$, Brambilla DJ, et al. Prevalence and incidence of androgen deficiency in middle-aged and elderly men: Estimates from the Massachusetts Male Aging Study. J Clin Endocrinol Metab 2004; 89:5920-5926.

22. Travison G, Araujo AB, Kupelian V, et al. The relative contributions of aging, health and lifestyle factors to serum testosterone decline in men. J Clin Endocrinol Metab 2007;92:549-555.

23. Araujo AB, Esche GR, Kupelian V, et al. Prevalence of symptomatic androgen deficiency in men. J Clin Endocrinol Metab 2007; 92:4241-4247.

24. Mulligan T, Frick MF, Zurow QC, et al. Prevalence of hypogonadism in males aged at least 45 years: The HIM Study. Int J Clin Pract 2006; 60:762-769.

25. Schneider HJ, Siewers C, Klotsche J, et al. Prevalence of low male testosterone levels in primary care in Germany: Cross sectional results from the DETECT study. Clin Endocrinol 2009; 70:446-454.

26. Biswas M, Hampton D, Newcombe RG, Rees DA. Total and free testosterone concentrations are strongly influenced by age and central obesity in men with typ 1 and type 2 diabetes but correlate weakly with symptoms of androgen deficiency and diabetes-related quality of life. Clin Endocrinol 2012; 76:665-673. 